\title{
Images of the month 1: Diabetic striatopathy without hemichorea/hemiballism
}

\author{
Authors: Choon-Bing Chua, ${ }^{\mathrm{A}}$ Hung-Cheng Chen, ${ }^{\mathrm{B}}$ Hung-Yuan Su, ${ }^{\mathrm{C}} \mathrm{I}-\mathrm{Ting}$ Tsai ${ }^{\mathrm{D}}$ and Cheuk-Kwan Sun ${ }^{\mathrm{E}}$
}
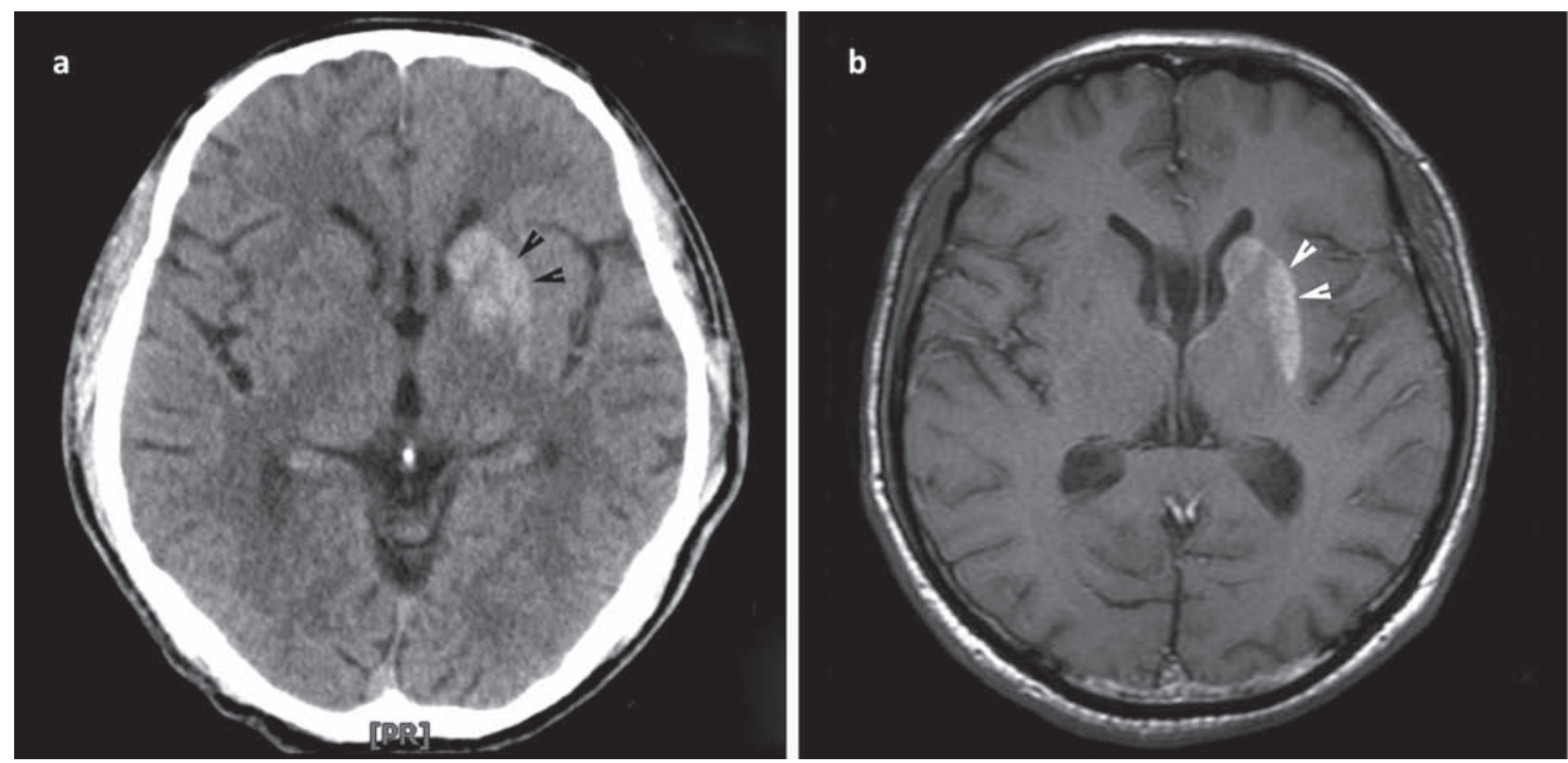

Fig 1. Typical imaging presentations of diabetic striatopathy. (a) Computed tomography showing hyperdensity in left basal ganglia (black arrows). (b) T1-weighted magnetic resonance imaging revealing hyperintensity in left basal ganglia (white arrows).

KEYWORDS: Basal ganglia syndrome, striatal hyperintensity, chorea, nonketotic hyperglycemia

\section{Case presentation}

A 53-year-old man with a history of poorly controlled diabetes was sent to the emergency department due to syncope with

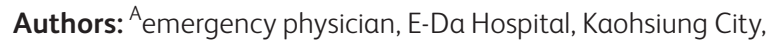
Taiwan and I-Shou University, Kaohsiung City, Taiwan; ${ }^{B}$ emergency physician, E-Da Hospital, Kaohsiung City, Taiwan and I-Shou University, Kaohsiung City, Taiwan; ${ }^{C}$ emergency physician, E-Da Hospital, Kaohsiung City, Taiwan and I-Shou University, Kaohsiung City, Taiwan; Demergency physician, E-Da Hospital, Kaohsiung City, Taiwan and I-Shou University, Kaohsiung City, Taiwan; ${ }^{E}$ emergency physician, E-Da Hospital, Kaohsiung City, Taiwan and I-Shou University, Kaohsiung City, Taiwan 10-minute conscious disturbance. On arrival, he looked fatigued but alert without any neurological deficit. His serum glucose and glycated haemoglobin concentrations were $645 \mathrm{mg} / \mathrm{dL}$ and $17.1 \%$, respectively. No urinary ketone was noted. An unenhanced computed tomography (CT) of the brain revealed hyperdensity in the left basal ganglia, with sparing of the internal capsule and no mass effect (Fig 1a). T1-weighted magnetic resonance imaging (MRI) demonstrated hyperintensity in the corresponding basal ganglia (Fig 1b). The patient was admitted for glucose control with the diagnosis of diabetic striatopathy.

The term 'diabetic striatopathy' (DS), introduced in 2009,' denoted a relatively uncommon hyperglycaemic condition associated with hemichorea/hemiballism relating to the contralateral reversible abnormality of basal ganglia on CT or/ and MRI, although it was described previously as hemichorea/ hemiballism. ${ }^{2-4}$ Only a few reported cases presented without hemichorea. ${ }^{5}$ The disease has been documented to occur predominantly in elderly Asian females with poorly controlled type 2 diabetes mellitus (DM). The chorea/ballism could manifest intermittently or continuously, and start abruptly or 
insidiously from low to high amplitude with sleep suppression in most reported cases. Control of hyperglycaemia often results in resolution of chorea/ballism as well as striatal abnormalities. Additional anti-chorea medication may be needed if the chorea is not suppressed by strict glucose control.

To date, regarding the pathogenesis of DS-associated striatal abnormalities on neuroimaging, there have been four hypotheses, namely, infarction with gemistocyte infiltration, mineral deposition, myelin destruction and microhaemorrhage. There were four pathology reports revealing evidence of haemorrhage including haemosiderin-containing macrophages, ${ }^{4}$ extravascular haemosiderin deposits, ${ }^{2}$ extravasated erythrocytes, ${ }^{1}$ and microhaemorrhage. ${ }^{3}$ Together with the reversible nature of the striatal anomaly, initial CT hyperdensity reflecting haemorrhage, high-signal intensity T1 weighted MRI corresponding to paramagnetic material, oozing around the basal ganglia on magnetic resonance angiography ${ }^{5}$ made microhaemorrhage a probability.

\section{References}

1 Abe Y, Yamamoto T, Soeda T et al. Diabetic striatal disease: clinical presentation, neuroimaging, and pathology. Internal Med 2009:48:1135-41.
2 Mestre TA, Ferreira J], Pimentel J. Putaminal petechial haemorrhage as the cause of non-ketotic hyperglycaemic chorea: a neuropathological case correlated with MRI findings. J Neurol Neurosurg Psychiatry 2007:78:549-50

3 Nath J, Jambhekar K, Rao C, Armitano E. Radiological and pathological changes in hemiballism-hemichorea with striatal hyperintensity. J Magn Reson Imaging 2006;23:564-8.

4 Ohara S, Nakagawa S, Tabata K, Hashimoto T. Hemiballism with hyperglycemia and striatal T1-MRI hyperintensity: an autopsy report. Movement Disord 2001;16:521-5.

5 Sato H, Hamano M, Fushimi E et al. Diabetic striatopathy manifesting as severe consciousness disturbance with no involuntary movements. Diabetic Med 2017:34:1795-9.

Address for correspondence: Prof Cheuk-Kwan Sun, No. 1 Yi-Da Road, Jiao-Su Village, Yan-Chao District, Kaohsiung City 824, Taiwan.

Email: lawrence.c.k.sun@gmail.com Treating tobacco dependency in the NHS

Published in 2018, this report by the Tobacco Advisory Group of the Royal College of Physicians addresses the harms and costs arising from smoking in the patients we see every day, and argues for a new approach to treating their addiction.

Download the report at: www.rcplondon.ac.uk/hidingplain-sight-treating-tobaccodependency-nhs

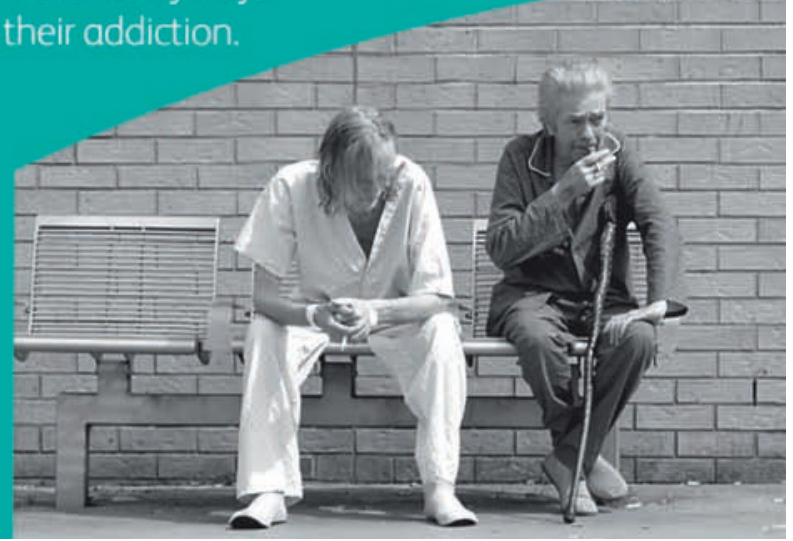

